\title{
Unitary classification in a comparison task
}

\author{
XIAOFENG LI and ALBERT F. SMITH \\ State University of New York, Binghamton, New York
}

\begin{abstract}
Sequential effects were used to diagnose whether elements in a two-object comparison task are represented as a perceptual unit or separately. The presence of sequential effects and absence of influences of individual elements on the subsequent trial in a successive comparison task favor the hypothesis that the elements in a pair are represented as a unit, and that a response is made to the perceptual unit. The patterns of response times on same and different trials differed in several ways; these suggested that the quality of the representations of same and different trials may differ.
\end{abstract}

Comparison is a fundamental human cognitive operation that involves deciding whether objects are the same on specified attributes. The comparison task has served as a standard method for studying cognitive issues that range from perceptual coding to concept learning (see the review by Farell, 1985). Generally, investigators who have either studied or used the comparison task have assumed that the objects that are to be compared are represented separately (although see, e.g., Krueger, 1973). In the present study, through analyses of sequential effects in response times, we explored the possibility that the tobe-compared objects are represented as a single perceptual unit. To clarify the rationale for these studies, we will first review two experimental tasks that are often used as tools in making inferences about the representation of stimulus structure. We will then define the sequential effects of interest and explain their relevance to the evaluation of stimulus representations. Finally, we will review two accounts of sequential effects in order to develop alternative sets of predictions for the experiments that we present here.

\section{Unconditional and Conditional Classification Tasks}

Psychologists have used various classification tasks to make inferences about the internal representation of stimulus structure (see Garner, 1974, 1988; Nickerson, 1972). In an unconditional classification task, the subject classifies stimulus displays according to a rule that is typically specified in terms of levels of a single stimulus property.

This research was supported by BRSG Grant S07RR07149-13 from the Biomedical Research Support Grant Program, Division of Research Resources, National Institutes of Health, to the Research Foundation of the State University of New York at Binghamton. Preparation of the manuscript was facilitated by the Center for Cognitive and Psycholinguistic Sciences. We thank C. M. Connine, A. W. Inhoff, R. E. Pastore, C. W. Eriksen, R. Proctor, and one anonymous reviewer for their valuable comments on earlier drafts of this paper, and J. Cole, R. Farber, and D. Sullivan for their assistance in conducting this research. Correspondence and requests for reprints should be sent to A. F. Smith, Department of Psychology, State University of New York, P.O. Box 6000, Binghamton, NY 13902-6000.
For example, subjects may classify stimuli according to their levels on a specified dimension such as shape or color. The term unconditional refers to the invariance of the mapping of stimuli to responses through any block of trials.

In a conditional classification task, as conventionally conceived, two stimulus properties are relevant on each trial: The subject classifies one stimulus property conditionally on the value of the other. Technically, a comparison task is a conditional classification task because, on each trial of a comparison experiment, the response to what is nominally the second object is conditional on one or more attributes of the first object.

According to the standard view of comparison trials, the observer treats as separate the two nominal objects that are presented (Proctor, 1981). We call this view the separate representation hypothesis. However, an alternative strategy is logically possible and, under certain circumstances, compellingly plausible (Krueger, 1973; A. F. Smith, 1986): The observer may treat the stimulus pair as a unit and classify the unit. For example, in a comparison experiment with four stimulus pairs-two same pairs and two different pairs-the observer may use an unconditional 4-to-2 mapping of stimulus displays to responses through an entire block of trials. Rather than compare the nominal objects within a pair, the observer may encode the objects of the pair as a perceptual unit, and then respond to a match between the unit and a memory representation of that unit. We call this the unitary representation hypothesis.

\section{Sequential Effects in Response Time Data}

To evaluate these two representational hypotheses as accounts of performance in successive comparison, we examined patterns of sequential effects in response times. Sequential effects are present in data when response times to a stimulus depend on the identity of (and, possibly, the response to) the preceding stimulus (Kirby, 1980; Kornblum, 1973; Luce, 1986, sections 6.6, 10.3).

In this article, we restrict our attention to situations in which four stimuli are mapped to two responses. Consider, for example, an unconditional classification task 
in which four letters- $x, X, y$, and $Y$-are to be classified by name, regardless of case. Three types of transition between trials are distinguishable according to the relation between the stimulus on a trial and the stimulus on the preceding trial: In identical transitions, a stimulus is preceded by itself (e.g., $\mathrm{X}$ preceded by $\mathrm{X}$ ); in equivalent transitions, a stimulus is preceded by the other stimulus that is mapped to the same response (e.g., $\mathrm{X}$ preceded by $\mathrm{X}$ ); in complementary transitions, a stimulus is preceded by a stimulus mapped to the other response (e.g., $\mathrm{X}$ preceded by $y$ or $Y$ ). Complementary transitions can be distinguished further according to whether the current stimulus differs from its predecessor in both attributes or in only the attribute relevant to classification. Our use of identical and equivalent is consistent with their previous usage in the literature on sequential effects (Bertelson, 1965; Kornblum, 1973; M. C. Smith, 1968); we substitute complementary for the previously used different to describe transitions to a stimulus that requires the alternate response. Studies of unconditional classification have shown that responses that follow identical transitions are faster than responses that follow equivalent transitions, which are often faster than those that follow complementary transitions (e.g., Felfoldy, 1974). We will focus primarily on comparisons of trial sequences over which response patterns are the same but specific stimuli are not.

\section{Accounts of Sequential Effects}

A complete discussion of explanations of sequential effects in response time would rove well beyond the scope of this article. However, to frame the ensuing discussion, we outline here two of the hypotheses that have been entertained.

Two sorts of automatic mechanisms have been given special consideration (see Kirby, 1980). The encoding facilitation explanation attributes faster responding on trials that follow identical rather than equivalent transitions to the priming, by the preceding stimulus, of neural pathways or representational nodes (Nickerson, 1973; Posner, Klein, Summers, \& Buggie, 1973). By this account, the amount of facilitation afforded to the processing of a stimulus on any trial should depend on the degree of representational overlap between that stimulus and the preceding stimulus. According to the encoding facilitation account, sequential effects should diminish as the time between two adjacent trials is increased, and it should be eliminated if a distractor stimulus is interposed between trials. However, observations of sequential effects at long responsestimulus intervals (RSIs) and when a distractor stimulus occurs during the RSI (Kroll \& Ramskov, 1984; Walker \& Marshall, 1982) indicate that the encoding facilitation account is not sufficient. The response repetition, or response priming, explanation attributes sequential effects to residual activation or priming of motor systems from the previous trial (e.g., Eriksen \& Schultz, 1979). According to this account, the response should be facilitated whenever the response to the current trial is a repetition of that of the preceding trial.
If two response classes are defined according to a single stimulus attribute, any response repetition is necessarily accompanied by at least partial stimulus repetition, because the response-determining aspect of the stimulus is repeated. However, stimulus and response repetition are decoupled in the comparison task, in which the response depends on the relation between a pair of stimulus objects, not on the identities of those objects (see also M. C. Smith, 1968). If the response is considered as being made to what is nominally - and, in the case of successive comparison, actually-the second object, then, in comparison, each response is made to every object; there are no consistent mappings of stimulus objects to responses (see also Krueger \& Shapiro, 1981). In this article, we report studies of sequential effects in successive comparison.

\section{Sequential Effects in Comparison Response Times}

Two nominal stimulus objects, $A$ and $B$, may be combined factorially to generate the four stimulus pairs-[AA], $[A B],[B A]$, and $[\mathrm{BB}]$ - that would be used in the simplest comparison experiment. Our concern in this paper is with successive comparison, so that, for example, [AA] denotes a trial on which Object $A$ is presented, followed by an empty interval of specified duration and then by a second presentation of Object A. A trial is defined as a same trial or as a different trial according to the correct response to the stimulus pair presented on that trial.

Table 1 shows the 16 sequences of stimulus pairs that could occur on two adjacent trials; these 16 sequences may be classified according to the type of transition to the current trial (trial $n$ ) from the preceding trial (trial $n-1$ ). For each same and different trial, there are four transition types represented by stimulus pairs presented on successive trials (i.e., [trial $n-1$ ] [trial $n$ ]) that are analogous to those described for the unconditional classification task. An identical transition occurs when a particular pair is preceded by itself (e.g., [AA][AA] or [AB][AB]). An equivalent transition occurs when a pair is preceded by the other pair that is mapped to its response (e.g., [BB][AA] or $[\mathrm{BA}][\mathrm{AB}])$. A complementary transition occurs when a pair is preceded by a pair that is mapped to the alternate response. We distinguish between complementary- 1 transitions, in which the second element of the trial $n-1$ pair differs from the first element of the trial $n$ pair (e.g., $[\mathrm{AB}][\mathrm{AA}]$ or $[\mathrm{BB}][\mathrm{AB}]$ ), and complementary-2 transitions, in which the second element of the trial $n-1$ pair is the same as the first element of the trial $n$ pair (e.g., [BA][AA] or $[A A][A B])$.

\section{Joint Predictions of the Hypotheses Concerning Representation and Mechanisms}

The principal goal in the present study was to use sequential effects to assess the adequacy of the two representational hypotheses. Because of the potential viability of several explanations for sequential effects-including encoding facilitation and response repetition-several sets of predictions are required. Predictions about the results of comparison experiments, assuming each combination 
Table 1

Possible Stimulus Sequences on Two Adjacent Trials

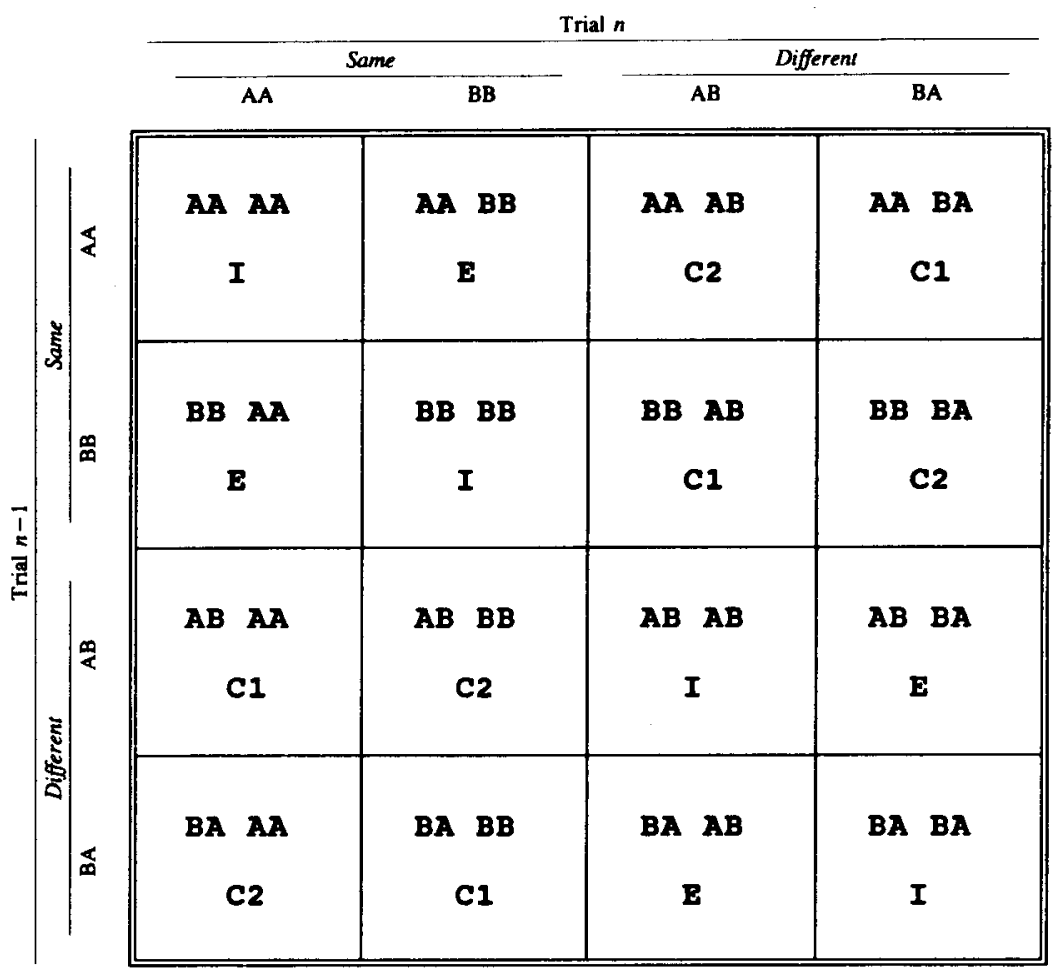

Note-In each cell of the matrix, a pair of letters represents a trial with two stimuli denoted by $A$ or B. Two pairs represent two adjacent trials. The letter below indicates the transition type: I, identical transition; E, equivalent transition; $\mathrm{C}$ 1, complementary-1 transition; $\mathrm{C}$, complementary-2 transition.

of mechanism and representational hypothesis, are outlined below and summarized in Table 2 .

If we assume a simple response repetition mechanism as the explanation of sequential effects, response times will depend solely on the relationship of the response on trial $n$ to the response on trial $n-1$. Thus, for neither same nor different trials should speeds of responses that follow equivalent transitions differ from the speeds of responses that follow identical transitions, and speeds of responses that follow complementary- 1 transitions should not differ from the speeds of responses that follow complementary- 2 transitions. These predictions do not depend on whether the nominal stimulus elements are represented individually or as a unit.

If encoding facilitation is responsible for sequential effects, predictions about experimental results depend on the nature of the stimulus representation that is assumed. If the stimulus elements of each trial are represented separately, encoding facilitation would be expected for individual elements. Thus, response times in a successive comparison experiment might depend in part on betweentrial encoding facilitation that stems from the relationship of the second element of trial $n-1$ to the first element of trial $n$. In particular, the encoding of the first element

Table 2

Predictions Concerning Sequential Effects (Given Representation and Mechanism)

\begin{tabular}{|c|c|c|c|c|c|}
\hline & \multirow{3}{*}{$\begin{array}{l}\text { Response } \\
\text { Repetition }\end{array}$} & \multicolumn{4}{|c|}{ Encoding Facilitation } \\
\hline & & \multicolumn{2}{|c|}{ Separate } & \multicolumn{2}{|c|}{ Unitary } \\
\hline & & Same & Different & Same & Different \\
\hline $\begin{array}{l}I \text { versus } E \\
C 1 \text { versus } C 2\end{array}$ & $\begin{aligned} \mathbf{R T}_{1} & =\mathrm{RT}_{\mathrm{E}} \\
\mathrm{RT}_{\mathrm{C} 1} & =\mathrm{RT}_{\mathrm{C} 2}\end{aligned}$ & $\begin{aligned} \mathrm{RT}_{1} & <\mathrm{RT}_{\mathrm{E}} \\
\mathrm{RT}_{\mathrm{C} 1} & >\mathrm{RT}_{\mathrm{C} 2}\end{aligned}$ & $\begin{aligned} \mathrm{RT}_{\mathrm{I}} & >\mathrm{RT}_{\mathrm{E}} \\
\mathrm{RT}_{\mathrm{C} 1} & >\mathrm{RT}_{\mathrm{C} 2}\end{aligned}$ & $\begin{aligned} \mathrm{RT}_{\mathrm{I}} & <\mathrm{RT}_{\mathrm{E}} \\
\mathrm{RT}_{\mathrm{Cl}} & =\mathrm{RT}_{\mathrm{C} 2}\end{aligned}$ & $\begin{aligned} \mathrm{RT}_{\mathrm{I}} & <\mathrm{RT}_{\mathrm{E}} \\
\mathrm{RT}_{\mathrm{Cl}} & =\mathrm{RT}_{\mathrm{C} 2}\end{aligned}$ \\
\hline
\end{tabular}


of trial $n$ may be facilitated by identity to the second element of trial $n-1$, and this may affect response times. For same trials, the stimulus elements of a trial that follows an identical transition are repetitions of the elements of the preceding trial (e.g., [AA] $[A A])$, but those of a trial that follows an equivalent transition are different (e.g., [BB][AA]). If an identity relation between the interior elements speeds processing, faster responses should follow identical than follow equivalent transitions. For different trials, however, the reverse pattern of response times is predicted: Following identical transitions, the first element of trial $n$ differs from the second element of trial $n-1$ (e.g., [AB][AB]), whereas following equivalent transitions, the first element of trial $n$ is the same as the second element of trial $n-1$ (e.g., [BA][AB]). Thus, given between-trial element-based encoding facilitation, responses that follow identical transitions should be slower than those that follow equivalent transitions. For both same and different trials, faster responses should follow complementary-2 transitions (e.g., [BA][AA] and [AA][AB]) than follow complementary-1 transitions (e.g., $[\mathrm{AB}][\mathrm{AA}]$ and $[\mathrm{BB}][\mathrm{AB}]$ ). (Recall that the two types of complementary transitions are distinguished according to the relationship between the second element of trial $n-1$ and the first element of trial $n$.)

If the two stimulus elements on any trial are represented as a unit, subjects would essentially be carrying out an unconditional classification task, and predictions about sequential effects would be based on the relationship of the entire stimulus pairs on successive trials. For both same and different trials, faster responses should be observed following identical transitions than following equivalent transitions, since the stimulus pair from the preceding trial is repeated following identical, but not following equivalent transitions. In addition, responses that follow complementary-1 transitions and complementary- 2 transitions should not differ in speed.

In the experiments reported below, we evaluated these predictions by studying sequential effects under manipulations of conditions designed to influence the likelihood that a pair of nominal stimuli would be treated as a unit (e.g., interstimulus interval-the time between to-becompared elements on a single trial) and the likelihood that the events of a trial would affect those of the next (e.g., intertrial interval-the time between trials).

\section{EXPERIMENT 1}

The first experiment was designed to determine whether trial-to-trial sequential effects are present in response time data from successive comparison tasks, and, if so, to permit preliminary insight into their origin.

If response repetition alone causes sequential effects, then, regardless of the nature of the stimulus representation, there should be no difference between speeds of responses that follow identical transitions and equivalent transitions, and no difference between speeds of responses that follow complementary-1 transitions and complemen- tary- 2 transitions. Thus, response time data that contained sequential effects due solely to response repetition would not help discriminate between the representational hypotheses. On the other hand, suppose that sequential effects are due to facilitation of stimulus encoding by the preceding stimulus. Even if the unitary representation hypothesis is valid, a two-element perceptual unit may disintegrate as the time between the two elements increases. If unconditional and conditional classification require different amounts of time, this would result in an interaction in response times between interstimulus interval (ISI) and transition type; the pattern of any dependence of response times on transition type should vary over ISIs. For example, if the perceptual unit disintegrates, then at very long ISIs, the pattern of response times as a function of transition types should resemble the pattern predicted assuming separate representations of elements (see Table 2).

\section{Method}

Subjects. Eight undergraduates participated to fulfill partially the research-exposure requirement of their introductory psychology course.

Design and Procedure. The stimulus elements were two lights (Dialco 507-3913; .26 M.S.C.P.), denoted here as A and B, mounted vertically on a panel that was located approximately at the subjects' eye level. The center-to-center distance of the lights was approximately $5 \mathrm{~cm}$, and the subjects viewed the panel, from a distance of approximately $1 \mathrm{~m}$, in an illuminated room.

The stimulus pairs were the four possible combinations of two lights illuminated in succession ([AA], [AB], [BA], and [BB]). On each trial, one light, illuminated for $500 \mathrm{msec}$, was followed by an ISI during which no light was illuminated and then by a second light, which was response-terminated. Three ISIs $(250,500$, and $1,000 \mathrm{msec}$ ) were used; each subject completed three blocks of 100 trials at each level of ISI. Blocks of trials were ordered randomly, with the constraint that no value of ISI be repeated in adjacent blocks. The experiment began with a block of 100 practice trials.

The subjects were instructed to respond according to whether the two illuminated lights were same or different, and to respond as rapidly as possible while minimizing errors. The subjects responded by pressing one of two buttons (Refac Model TC-1/MF), each about $3 \mathrm{~cm}$ in diameter, mounted side by side on a Plexiglas plate at a center-to-center distance of $3.2 \mathrm{~cm}$. A travel of about $0.01 \mathrm{~cm}$ is required to set these switches. Response times were measured to the nearest $5 \mathrm{msec}$ from the onset of the second stimulus element. Each trial began $2 \mathrm{sec}$ after a response. Only latencies of correct responses that followed correct responses were analyzed.

\section{Results}

The average error rate across subjects was .07; values for individual subjects ranged from .006 to .082 . The relationship between accuracy and response speed was assessed prior to carrying out analyses for treatment effects, by computing, for each subject over blocks of trials, the correlation between the error rate and mean response times. Over subjects, the average correlation between the error rate and response times was -.16 , indicating that the subjects did not trade accuracy for speed.

Table 3 shows, for same and different trials, for each combination of ISI and transition type, the mean of median response times, its standard error, and the mean error rate. Table 4 summarizes the test statistics for separate 4 (tran- 
Table 3

Mean Response Times (in Milliseconds), Standard Errors, and Mean Error Rate in Experiment 1

\begin{tabular}{|c|c|c|c|c|c|c|c|c|}
\hline \multirow{3}{*}{$\begin{array}{c}\text { Transition } \\
\text { Type }\end{array}$} & \multicolumn{8}{|c|}{ Interstimulus Interval } \\
\hline & \multicolumn{2}{|c|}{250} & \multicolumn{2}{|c|}{500} & \multicolumn{2}{|c|}{1,000} & \multirow[b]{2}{*}{ Mean } & \multirow{2}{*}{$\begin{array}{c}\text { Error } \\
\text { Rate }(\%)\end{array}$} \\
\hline & $M$ & $S E$ & $M$ & $S E$ & $M$ & $S E$ & & \\
\hline \multicolumn{9}{|c|}{ Same Trials } \\
\hline $\mathbf{I}$ & 386 & 19 & 438 & 28 & 454 & 36 & 426 & 3.4 \\
\hline $\mathbf{E}$ & 420 & 29 & 469 & 40 & 465 & 36 & 451 & 5.7 \\
\hline $\mathrm{Cl}$ & 476 & 28 & 501 & 36 & 555 & 46 & 511 & 7.1 \\
\hline $\mathrm{C} 2$ & 467 & 28 & 507 & 36 & 537 & 46 & 504 & 11.0 \\
\hline \multicolumn{9}{|c|}{ Different Trials } \\
\hline I & 430 & 34 & 469 & 42 & 516 & 41 & 472 & 6.3 \\
\hline E & 484 & 34 & 499 & 36 & 552 & 55 & 512 & 7.1 \\
\hline $\mathrm{C} 1$ & 454 & 40 & 468 & 49 & 535 & 75 & 486 & 7.7 \\
\hline $\mathrm{C} 2$ & 417 & 33 & 448 & 36 & 482 & 52 & 449 & 4.8 \\
\hline
\end{tabular}

Note-I, identical transition; E, equivalent transition; C1, complementary-1 transition; $\mathrm{C} 2$, complementary-2 transition. Interstimulus intervals are given in milliseconds.

sition types) $\times 3$ (ISIs) analyses of variance conducted for same and different trials. The significant main effects of transition type indicate the presence of sequential effects. Planned comparisons showed, for both same and different trials, that responses to trials that followed identical transitions were significantly faster than responses to trials that followed equivalent transitions. For neither same nor different trials did speeds of responses to trials that followed complementary-1 transitions differ significantly from speeds of those that followed complementary- 2 transitions. The ISI did not influence the pattern of sequential effects: Although response times increased as ISI increased for both same and different trials, the interaction of ISI and transition type was not significant for either type of trial.

\section{Discussion}

These data show that intertrial sequential effects occur in successive comparison tasks. As summarized in Table 2, the response repetition mechanism suggests that response times should be equal following identical and equivalent transitions, regardless of the nature of the internal representation of the stimulus display. The results are clearly inconsistent with this prediction and mandate rejection of response repetition as an exclusive account of sequential effects.

The results of Experiment 1 are generally consistent with the predictions of the unitary representation hypoth-

Table 4

Test Statistics for Experiment 1

\begin{tabular}{lccc}
\hline $\begin{array}{c}\text { Source } \\
\text { of Variance }\end{array}$ & $d f$ & $\begin{array}{c}\text { Same } \\
F\end{array}$ & $\begin{array}{c}\text { Different } \\
F\end{array}$ \\
\hline Transition & 3,21 & $19.19^{*}$ & $5.72^{*}$ \\
ISI & 2,14 & $7.25^{*}$ & $9.03^{*}$ \\
Transition $\times$ ISI & 6,42 & 1.11 & 0.49 \\
I versus E & 1,7 & $5.65^{*}$ & $35.47^{*}$ \\
C1 versus C2 & 1,7 & 0.43 & 3.88 \\
\hline
\end{tabular}

Note-ISI, interstimulus interval; I, identical transition; E, equivalent transition; $\mathrm{C1}$, complementary-1 transition; $\mathrm{C2}$, complementary-2 transition. ${ }^{*} p<.05$. esis, assuming encoding facilitation. For both same and different trials, responses were faster following identical than following equivalent transitions. In addition, for neither same nor different trials did response times depend significantly on type of complementary transition. These results are inconsistent with the supposition that the individual elements of the stimulus presentation of a trial affect performance on the subsequent trial, and suggest instead that the stimulus pair presented on a trial is represented as a perceptual unit. The absence of an interaction between transition type and ISI is somewhat troubling; evidently time alone, in amounts bounded by the longest ISI used in this study, did not effectively cause the representational units to disintegrate (see also Luce, Nosofsky, Green, \& Smith, 1982).

The error data shown in Table 3 indicate that same and different trials exert different influences on subsequent trials. On same trials, more errors followed complementary transitions than followed identical or equivalent transitions, whereas the opposite pattern characterized the data of different trials. The implications of this pattern will be discussed in the context of Experiment 2, in which stimulus variables were manipulated to evaluate the plausibility of encoding facilitation as the mechanism responsible for the sequential effects observed in this comparison task.

\section{EXPERIMENT 2}

Kornblum (1973) reviewed evidence that sequential effects deteriorate as RSI is lengthened and argued that the dependence of the magnitude of sequential effects in response times on RSI suggests the operation of an encoding facilitation mechanism. In Experiment 2, we investigated the impact of variation in RSI on sequential effects in successive comparison.

\section{Method}

Subjects. Eight undergraduates participated to fulfill partially the research-exposure requirement of their introductory psychology course. 
Table 5

Mean Response Times (in Milliseconds), Standard Errors, and Mean Error Rate in Experiment 2

\begin{tabular}{|c|c|c|c|c|c|c|c|c|c|c|c|c|c|c|}
\hline \multirow{3}{*}{$\begin{array}{c}\text { Transition } \\
\text { Type }\end{array}$} & \multicolumn{4}{|c|}{ RSSI 200} & \multicolumn{4}{|c|}{ RSI 1,000} & \multicolumn{4}{|c|}{ RSI 2,000} & \multirow[b]{3}{*}{ Mean } & \multirow{3}{*}{$\begin{array}{c}\text { Error } \\
\text { Rate (\%) }\end{array}$} \\
\hline & \multicolumn{2}{|c|}{ ISI 100} & \multicolumn{2}{|c|}{ ISI 1,000} & \multicolumn{2}{|c|}{ ISI 100} & \multicolumn{2}{|c|}{ ISI 1,000} & \multicolumn{2}{|c|}{ ISI 100} & \multicolumn{2}{|c|}{ ISI 1,000} & & \\
\hline & $M$ & $S E$ & $M$ & $S E$ & $M$ & $S E$ & $M$ & $S E$ & $M$ & $S E$ & $M$ & $S E$ & & \\
\hline \multicolumn{15}{|c|}{ Same Trials } \\
\hline I & 356 & 20 & 451 & 27 & 392 & 16 & 413 & 12 & 385 & 14 & 416 & 17 & 402 & 0.6 \\
\hline $\mathrm{E}$ & 405 & 27 & 461 & 32 & 403 & 12 & 446 & 20 & 408 & 16 & 436 & 17 & 427 & 3.9 \\
\hline $\mathrm{Cl}$ & 401 & 37 & 506 & 45 & 417 & 13 & 490 & 20 & 427 & 13 & 460 & 18 & 450 & 4.6 \\
\hline $\mathrm{C} 2$ & 418 & 23 & 508 & 36 & 419 & 16 & 486 & 19 & 430 & 13 & 459 & 18 & 453 & 4.5 \\
\hline \multicolumn{15}{|c|}{ Different Trials } \\
\hline I & 435 & 32 & 497 & 28 & 434 & 17 & 473 & 17 & 451 & 15 & 494 & 17 & 464 & 3.6 \\
\hline $\mathrm{E}$ & 534 & 61 & 610 & 60 & 470 & 19 & 507 & 22 & 455 & 18 & 498 & 23 & 512 & 7.3 \\
\hline $\mathrm{Cl}$ & 423 & 36 & 492 & 30 & 430 & 18 & 475 & 26 & 464 & 18 & 469 & 20 & 459 & 3.7 \\
\hline $\mathrm{C} 2$ & 518 & 71 & 449 & 34 & 452 & 15 & 439 & 17 & 455 & 17 & 460 & 17 & 462 & 3.9 \\
\hline
\end{tabular}

Note-RSI, response-stimulus interval; ISI, interstimulus interval; I, identical transition; E, equivalent transition; Cl, complementary-1 transition; C2, complementary-2 transition. RSIs and ISIs are given in milliseconds.

Design and Procedure. Three levels of RSI (200, 1,000, and $2,000 \mathrm{msec})$ were factorially combined with two levels of ISI (100 and $1,000 \mathrm{msec}$ ) to create six experimental conditions. Subjects completed three blocks of 100 trials for each condition. These 18 blocks of trials were presented during two sessions on consecutive days. The subjects began each session with a block of 100 practice trials. The blocks were ordered randomly, with the constraint that two blocks of a single condition never be adjacent.

\section{Results}

The average error rate across subjects was .04; individual error rates ranged from .012 to .047 . Averaged over subjects, the correlation over blocks between the error rates and mean response times was .22, indicating that the subjects did not trade accuracy for speed.

Table 5 shows, for each experimental condition, the mean of median response times, its standard error, and the mean error rate. Separate 4 (transition types) $\times 3$ (RSIs) $\times 2$ (ISIs) within-subject analyses of variance were conducted for same and different trials. The test statistics are shown in Table 6. Significant main effects of transition type were found for both same and different trials. Planned comparisons showed, for both same and different trials, that responses that followed identical transitions were significantly faster than responses that followed equivalent transitions. For neither type of trial did response times following complementary-1 transitions differ significantly from those following complementary- 2 transitions. The overall pattern is consistent with the results of Experiment 1.

For same trials, neither RSI nor ISI influenced the pattern of sequential effects: Neither the transition type $x$ RSI interaction nor the transition type $\times$ ISI interaction was significant. The absence of an interaction between transition type and ISI for same trials, consistent with the results of Experiment 1, indicates that time alone is insufficient to break up representations of trials.

In contrast, for different trials, both of these interactions were significant. The interaction of transition type and RSI was due to a decrease in the magnitude of the sequential effects as RSI was lengthened: Responses that followed identical transitions were significantly faster than those that followed equivalent transitions when the RSI was $200 \mathrm{msec}$ $[F(1,7)=5.25, p<.05]$, but not when the RSI was $2,000 \mathrm{msec}[F(1,7)=.10]$. The interaction of transition type with ISI was due to a reversal, over the ISIs, in the relative speeds of responses that followed the two types of complementary transitions. At the short ISI (100 msec), responses that followed complementary-1 transitions were faster than those that followed complementary- 2 transitions, whereas at the long ISI $(1,000 \mathrm{msec})$, the opposite was observed.

\section{Discussion}

These results confirmed and extended the findings of Experiment 1, but they also suggested that a more complex account is required. First, as was true in Experiment 1 , for both same and different trials, response times were, on average, faster following identical than following equivalent transitions. Second, for neither same nor different trials did response times differ overall for the two types of complementary transitions. Additionally, for same trials, the patterns of response times over the several transition types depended neither on RSI nor on ISI.

The results for same trials are therefore consistent with the predictions of the unitary representation hypothesis. Subjects appear to have perceived the two successively presented elements as a unit. The apparent independence

Table 6

Test Statistics for Experiment 2

\begin{tabular}{lccc}
\hline & & Same & Different \\
Source of Variance & $d f$ & $F$ & $F$ \\
\hline Transition & 3,21 & $13.51^{*}$ & $11.60^{*}$ \\
ISI & 1,7 & $31.69^{*}$ & $7.77^{*}$ \\
RSI & 2,14 & 0.09 & 0.78 \\
Transition X ISI & 3,21 & 0.09 & $12.11^{*}$ \\
Transition X RSI & 6,42 & 0.14 & $5.22^{*}$ \\
Three-way interaction & 6,42 & 2.15 & $2.34^{*}$ \\
I versus E & 1,7 & $7.15^{*}$ & $11.47^{*}$ \\
C1 versus C2 & 1,7 & 0.16 & 0.22 \\
\hline
\end{tabular}

Note-ISI, interstimulus interval; RSI, response-stimulus interval; I, identical transition; E, equivalent transition; $\mathrm{C1}$, complementary-1 transition; $\mathrm{C} 2$, complementary-2 transition. $\quad{ }^{*} p<.05$. 
of transition types and RSI suggests that these unitary representations were stable over RSIs of as long as $2 \mathrm{sec}$.

The results for different trials are less favorable to the simple version of the unitary representation hypothesis that we have outlined. First, for different trials, but not for same trials, a significant interaction between transition type and ISI was observed. This interaction-due to a difference for pairs presented with different ISIs in the relative speeds of responses that followed the two complementary transitions-is not consistent with any of the predictions that we have described. We address this result in the General Discussion. Second, for different trials, but not for same trials, sequential effects diminished as RSI was increased. This result may be due to differences in stability over time between the representations of same and different trials, with the former being more stable than the latter.

This conjecture concerning differences in the stability of stimulus-pair representations is supported by the results of Experiments 1 and 2. For trials of each type in each experiment, the mean latency of responses to trials that followed identical and equivalent transitions was contrasted with the mean latency of responses to trials that followed complementary- 1 and complementary- 2 transitions. For same trials, responses that followed identical and equivalent transitions were significantly faster than those that followed complementary-1 and complementary-2 transitions [for Experiment 1, the means were 439 and $508 \mathrm{msec}$, respectively, $F(1,21)=54.71, p<.001$; for Experiment 2, the means were 415 and 452 msec, respectively, $F(1,21)=33.5, p<.001]$. However, for different trials, responses that followed identical and equivalent transitions were, on average, slower than those that followed complementary-1 and complementary- 2 transitions [for Experiment 1, the means were 492 and $468 \mathrm{msec}$, respectively, $F(1,21)=5.02, p<.05$; for Experiment 2, the means were 488 and $461 \mathrm{msec}$, respectively, $F(1,21)=$ $13.5, p<.01]$. In other words, responses to both same and different trials were faster following same trials than following different trials. Relative to same trials, different trials appear to interfere with processing of the subsequent trial. A general disruptive effect of different trials on subsequent performance is a consistent finding of investigators who have examined between-trials effects in comparison experiments (Krueger, 1983; Krueger \& Shapiro, 1981; Neill, Lissner, \& Beck, 1990).

The pattern of the error data shown in Table 5 was consistent with the pattern of response times in Experiment 2 and with the response time and error data of Experiment 1. For same trials, fewer errors followed identical and equivalent transitions than followed complementary transitions (on average, $2.3 \%$ and $4.6 \%$ error rates, respectively). For different trials, more errors followed identical and equivalent transitions than followed complementary transitions (on average, $5.5 \%$ and $3.8 \%$ error rates, respectively). In sum, fewer errors were made on trials that followed same trials than on trials that followed different trials. If internal representations of same trials are more stable than representations of different trials, they may interfere less with subsequent trials than do representations of different trials.

The results of Experiment 2 are generally consistent with the predictions of the unitary representation hypothesis. However, the simplicity of an account of sequential effects based purely on encoding facilitation of element pairs was compromised by the difference between same and different trials in the sensitivity of sequential effects to variation in RSI, and by the disparity in response times for different trials that followed the two types of complementary transitions.

\section{EXPERIMENT 3}

The stimuli used in the preceding experiments-highly discriminable spatial locations-were chosen for their simplicity. Yet spatial position of a visual stimulus has not been used typically as the relevant attribute in comparison experiments. To evaluate the generality of the results that have been described, Experiment 3 was conducted to examine intertrial sequential effects in comparisons of the frequencies of auditory signals. If elements presented for comparison are generally perceived as units and classified unconditionally, a pattern of results similar to that observed in Experiments 1 and 2 should be observed.

\section{Method}

Subjects. Fifteen undergraduate students participated in this experiment to satisfy partially the research-exposure requirement of their introductory psychology course. The data from 5 additional subjects were not analyzed, because their error rates either exceeded .10 or varied considerably over blocks of trials.

Design and Procedure. The two stimulus tones were square-wave signals of 880 and $990 \mathrm{~Hz}$, respectively, generated by a programmable sound generator (General Instrument AY-3-8910A). The subjects listened to tones at $70 \mathrm{~dB}(\mathrm{~A})$ via headphones (Radio Shack Realistic Nova-40) in a quiet room. On each trial, the first tone, presented for $500 \mathrm{msec}$, was followed by a silent ISI and then by a second tone which was response-terminated. Two ISIs (250 and $1,000 \mathrm{msec}$ ) were used; these occurred in alternate blocks of trials. The RSI was always $2 \mathrm{sec}$. Other details of the procedure were as those for Experiment 1. Each subject completed five blocks of $\mathbf{8 0}$ trials at each level of ISI; the first pair of trial blocks was considered practice and was not analyzed.

\section{Results}

Table 7 shows, for same and different trials, for each combination of ISI and transition type, the mean of median response times, its standard error, and the mean error rate. Separate 4 (transition types) $\times 2$ (ISIs) analyses of variance were conducted for same and different trials. Table 8 summarizes the test statistics from these analyses. The significant main effect of transition type indicates the presence of sequential effects in the comparison task with auditory signals. Planned comparisons showed, for both 
Table 7

Mean Response Times (in Milliseconds), Standard Errors, and Mean Error Rate in Experiment 3

\begin{tabular}{|c|c|c|c|c|c|c|}
\hline \multirow{3}{*}{$\begin{array}{c}\text { Transition } \\
\text { Type } \\
\end{array}$} & \multicolumn{4}{|c|}{ Interstimulus Interval } & \multirow[b]{3}{*}{ Mean } & \multirow{3}{*}{$\begin{array}{c}\text { Error } \\
\text { Rate (\%) }\end{array}$} \\
\hline & \multicolumn{2}{|c|}{$250 \mathrm{msec}$} & \multicolumn{2}{|c|}{$1,000 \mathrm{msec}$} & & \\
\hline & $M$ & $S E$ & $M$ & $S E$ & & \\
\hline \multicolumn{7}{|c|}{ Same Trials } \\
\hline I & 416 & 22 & 423 & 18 & 420 & 3.3 \\
\hline $\mathbf{E}$ & 467 & 30 & 466 & 22 & 467 & 4.3 \\
\hline $\mathrm{Cl}$ & 480 & 26 & 481 & 26 & 481 & 3.5 \\
\hline $\mathrm{C} 2$ & 475 & 28 & 481 & 24 & 478 & 4.5 \\
\hline \multicolumn{7}{|c|}{ Different Trials } \\
\hline I & 448 & 22 & 460 & 18 & 454 & 4.2 \\
\hline $\mathbf{E}$ & 479 & 27 & 490 & 21 & 485 & 4.3 \\
\hline $\bar{C} 1$ & 477 & 28 & 483 & 25 & 480 & 3.4 \\
\hline $\mathrm{C} 2$ & 428 & 23 & 422 & 23 & 425 & 2.3 \\
\hline
\end{tabular}

Note-I, identical transition; E, equivalent transition; C1, complementary-1 transition; $\mathbf{C 2}$, complementary-2 transition. Interstimulus intervals are given in milliseconds.

same and different trials, that responses that followed identical transitions were significantly faster than responses that followed equivalent transitions. For same trials, response times that followed complementary-1 transitions did not differ significantly from response times that followed complementary-2 transitions. However, for different trials, responses that followed complementary-2 transitions (e.g., $[\mathrm{AA}][\mathrm{AB}])$ were faster than responses that followed complementary-1 transitions (e.g., [BB][AB]). For neither same nor different trials did the pattern of sequential effects depend on ISI.

For same trials, responses that followed identical and equivalent transitions (444 $\mathrm{msec}$ ) were, on average, faster than responses that followed complementary-1 and complementary-2 transitions (480 msec) $[F(1,7)=15.47$, $p<.002]$. For different trials, however, responses that followed identical and equivalent transitions $(470 \mathrm{msec})$ were slower than responses that followed complementary1 and complementary- 2 transitions (453 msec) $[F(1,7)=$ $8.85, p<.01]$. Thus, overall, as was the case with the results of Experiments 1 and 2, responses that followed same trials were faster than responses that followed different trials.

\section{Discussion}

The principal features of the results are strikingly similar to those of Experiments 1 and 2. For same trials, the

Table 8

Test Statistics for Experiment 3

\begin{tabular}{lccc}
\hline \multicolumn{1}{c}{$\begin{array}{c}\text { Source } \\
\text { of Variance }\end{array}$} & $d f$ & Same & Different \\
\hline Transition & 3,42 & $14.80^{*}$ & $18.91^{*}$ \\
ISI & 1,14 & 0.12 & 0.33 \\
Transition $\times$ ISI & 3,42 & 0.16 & 1.48 \\
I versus E & 1,14 & $20.07^{*}$ & $12.47^{*}$ \\
C1 versus C2 & 1,14 & 0.13 & $30.92^{*}$ \\
\hline
\end{tabular}

Note-ISI, interstimulus interval; I, identical transition; E, equivalent transition; $\mathrm{C} 1$, complementary-1 transition; $\mathrm{C} 2$, complementary-2 transition. $* p<.05$. presence of intertrial sequential effects and the absence of influences of individual elements on the subsequent trial suggest that subjects treat a pair of stimulus elements-in Experiment 3, tones-as a perceptual unit (see Table 2). For different trials, the significant advantage of identical transitions over equivalent transitions in speeds of responses is consistent with the notion that pairs of elements in comparison tasks are treated as units. However, the results for different trials diverged in one salient way from the predictions of the unitary representation hypothesis: Responses that followed complementary- 2 transitions were faster than responses that followed complementary-1 transitions. We discuss this result in the General Discussion.

Consistent with the results of Experiments 1 and 2, different trials appeared to exert a general disruptive effect on the subsequent trial relative to same trials (see also Krueger, 1983; Krueger \& Shapiro, 1981; Neill et al., 1990). This suggests that the quality of stimulus representations on trials that follow different trials is inferior to the quality of stimulus representations on trials that follow same trials.

Since we excluded from analyses the data of subjects whose error rates were excessive, the pattern of errors in Experiment 3 is less clear than the error pattern in Experiments 1 and 2 . However, the general pattern is consistent with the results of those experiments. For same trials, the mean error rate on trials that followed identical and equivalent transitions was lower than the mean error rate on trials that followed complementary transitions; for different trials, the reverse was true. As discussed earlier, this pattern of error data is consistent with the hypotheses that the representations of same and different trials differ in quality and that different trials disrupt processing on subsequent trials.

\section{GENERAL DISCUSSION}

Intertrial sequential effects like those found in unconditional classification tasks occur in successive comparison as well. In general, elements of a stimulus pair presented for comparison do not individually influence performance on the subsequent trial. Rather, the results of the present experiments tend to support the unitary representation hypothesis-people appear to treat the stimulus pair as a unit.

Across the three experiments, certain consistencies in the results were independent of the type of stimuli used. For same trials, responses that followed identical transitions were faster than responses that followed equivalent transitions. In addition, speeds of responses that followed the two types of complementary transitions did not differ. These sequential effects did not depend on either RSI or ISI. For different trials, faster responses followed identical transitions than equivalent transitions in each of Experiments 1,2 , and 3 , although this advantage diminished as RSI was increased. This result suggested that representations of same trials are qualitatively superior to those of different trials. Although the effects of ISI on transition type for different trials were not completely consis- 
tent over experiments, no set of results was consistent with the predictions of the separate representation hypothesis. The pattern of response times on trials that followed complementary transitions varied over experiments and will receive a more elaborate discussion.

The pattern of performance for same and different trials differed in three cases in the experiments reported in this paper. First, sequential effects depended on RSI for different trials, but not for same trials. Second, for different trials, responses that followed complementary transitions were significantly faster than responses that followed identical and equivalent transitions, whereas for same trials, responses that followed complementary transitions were slower than those that followed identical and equivalent transitions. This discrepancy can, of course, be resolved by noting that, for both same and different trials, responses were faster following same trials than they were following different trials. In addition, the mean error rate was higher on trials that followed different trials than it was on trials that followed same trials. Third, when ISIs were at least $250 \mathrm{msec}$, "different" responses that followed complementary-2 transitions were faster than those that followed complementary-1 transitions. This last result was a decided tendency in the results of Experiment 1; it contributed to the significant transition type $\times$ ISI interaction in Experiment 2; and it was a significant effect in Experiment 3 . In contrast, for same trials, responses that followed complementary transitions did not depend, in any experiment, on the particular complementary transition involved.

These differences in the pattern of results for same and different trials suggest that the perceptual representations of same and different trials differ in quality. The representations of same trials may be more stable than those of different trials. Stable representations of same trials would be expected to exert consistent, specific effects on subsequent trials over longer periods of time than would the less stable representations of different trials; unstable, deteriorating representations of different trials would be expected to have general deleterious effects on performance on subsequent trials.

This account is similar to aspects of Krueger's (1978) "noisy-operator" model that included, as a central proposition, that internal noise and its effects on stimulus representation are important to performance in comparison tasks. Krueger proposed that the stimulus presentation on a trial has aftereffects that contribute to the noise level of the following trial. If a different trial generates more noise than does a same trial, Krueger's model may account for the results that we have reported. First, if different trials are noisier than same trials, the representations of different trials may be sufficiently degraded at long RSIs to explain why sequential effects for different trials deteriorate at long RSIs. Second, if different trials contribute more noise than same trials to the trials that follow them, then responses to any type of trial should be both slower and less accurate following different trials than following same trials.
The general tendency for "different" responses that followed complementary-2 transitions to be faster than those that followed complementary- 1 transitions suggests that the perception and processing of these stimulus pairs is more complicated than is contemplated by any of the schemes with which we began. For clarity, we emphasized exclusive positions-that responding to a stimulus pair might be facilitated by response repetition or pair repetition or identity between the second element of trial $n-1$ and the first element of trial $n$. If the influence of any trial on the subsequent trial is a weighted combination of these three effects, an account that weights pair repetition most heavily can be developed for the result, observed over experiments at ISIs greater than $250 \mathrm{msec}$, that "different" responses that follow complementary-2 transitions are faster than "different" responses that follow complementary-1 transitions. (This account would also accommodate the results of the three other pairwise comparisons-identical vs. equivalent for both same and different trials, and complementary-2 vs. complementary-1 for same trials.) Without additional data, we are not prepared to speculate about the relatively faster responses that followed complementary-1 transitions as opposed to complementary-2 transitions given very short ISIs.

In sum, we favor the notion that pairs as units contribute strongly to the results we have observed. First, the presence of sequential effects resembling those observed in unconditional classification tasks implicates the use of an unconditional classification strategy in this comparison task. Second, the consistent finding that responses that followed identical transitions were faster than responses that followed equivalent transitions is a central prediction of the unitary representation hypothesis. Finally, the prediction that response times should be independent of types of complementary transitions was observed consistently for same trials.

\section{REFERENCES}

Bertelson, P. (1965). Serial choice reaction time as a function of response versus signal-and-response repetition. Nature, 206, 217-218.

Eriksen, C. W., Schultz, D. W. (1979). Information processing in visual search: $A$ continuous flow conception and experimental results. Perception \& Psychophysics, 25, 249-263.

FARELL, B. (1985). "'Same"-" different" judgments: A review of current controversies in perceptual comparisons. Psychological Bulletin, 98, 419-456.

FeLfoldy, G. L. (1974). Repetition effects in choice reaction time to multidimensional stimuli. Perception \& Psychophysics, 15, 453-459.

GARNER, W. R. (1974). The processing of information and structure. Potomac, MD: Erlbaum.

GARNER, W. R. (1988). Facilitation and interference with a separable redundant dimension in stimulus comparison. Perception \& Psychophysics, 44, 321-330.

KIRBY, N. (1980). Sequential effects in choice reaction time. In A. T. Welford (Ed.), Reaction times (pp. 129-172). London: Academic Press.

KoRnBlum, S. (1973). Sequential effects in choice reaction time: A tutorial review. In S. Kornblum (Ed.), Attention and performance IV (pp. 256-288). NY: Academic Press.

Kroll, N. E., \& Ramskov, C. B. (1984). Visual memory as measured by classification and comparison tasks. Joumal of Experimental Psychology: Leaming, Memory, \& Cognition, 10, 395-420. 
KRUEGER, L. E. (1973). Effects of stimulus frequency on speed of "same"-" different" judgments. In S. Kornblum (Ed.), Attention and performance IV (pp. 497-506). New York: Academic Press.

KRUEger, L. E. (1978). A theory of perceptual matching. Psychological Review, 85, 278-304.

Krueger, L. E. (1983). Probing Proctor's priming principle: The effect of simultaneous and sequential presentation on same-different judgments. Joumal of Experimental Psychology: Learning, Memory, \& Cognition, 9, 511-523.

KruEger, L. E., Shapiro, R. G. (1981). Intertrial effects of samedifferent judgments. Quarterly Journal of Experimental Psychology, 33A, 241-265.

LUCE, R. D. (1986). Response times: Their role in inferring elementary mental organization. New York: Oxford University Press.

LuCE, R. D., Nosofsky, R. M., Green, D. M., \& SMTth, A. F. (1982). The bow and sequential effects in absolute identification. Perception \& Psychophysics, 32, 397-408.

NeILL, W. T., LissNer, L. S., \& BECK, J. L. (1990). Negative priming in same-different matching: Further evidence for a central locus of inhibition. Perception \& Psychophysics, 48, 398-400.

NiCKERSON, R. S. (1972). Binary-classification reaction time: A review of some studies of human information-processing capabilities. Psychonomic Monograph Supplements, 4(17, Whole No. 65), 275-317.

NiCKERSON, R. S. (1973). The use of binary-classification tasks in the study of human information processing: A tutorial survey. In S. Kornblum (Ed.), Attention and performance $I V$ (pp. 449-475). New York: Academic Press.

Posner, M. I., Klein, R., Summers, J., Buggie, S. (1973). On the selection of signals. Memory \& Cognition, 1, 2-12.

Proctor, R. W. (1981). A unified theory for matching-task phenomena. Psychological Review, 88, 291-326.

SмIтH, A. F. (1986). Strategies and structure in selective attention tasks. Unpublished doctoral dissertation, Yale University, New Haven, CT. SMITH, M. C. (1968). Repetition effect and short-term memory. Journal of Experimental Psychology, 77, 435-439.

Walker, P., Marshall, E. (1982). Visual memory and stimulus repetition effects. Joumal of Experimental Psychology: General, 111, 348-368.

(Manuscript received December 3, 1990; revision accepted for publication October 23, 1991.) 